\title{
Collective excitations of a degenerate gas at the BEC-BCS crossover
}

\author{
M. Bartenstein, ${ }^{1}$ A. Altmeyer, ${ }^{1}$ S. Riedl,${ }^{1}$ S. Jochim,${ }^{1}$ C. Chin,${ }^{1}$ J. Hecker Denschlag, ${ }^{1}$ and R. Grimm ${ }^{1,2}$ \\ ${ }^{1}$ Institut für Experimentalphysik, Universität Innsbruck, Technikerstraße 25, 6020 Innsbruck, Austria \\ ${ }^{2}$ Institut für Quantenoptik und Quanteninformation, \\ Österreichische Akademie der Wissenschaften, 6020 Innsbruck, Austria
}

(Dated: July 13, 2018)

\begin{abstract}
We study collective excitation modes of a fermionic gas of ${ }^{6} \mathrm{Li}$ atoms in the BEC-BCS crossover regime. While measurements of the axial compression mode in the cigar-shaped trap close to a Feshbach resonance confirm theoretical expectations, the radial compression mode shows surprising features. In the strongly interacting molecular BEC regime we observe a negative frequency shift with increasing coupling strength. In the regime of a strongly interacting Fermi gas, an abrupt change in the collective excitation frequency occurs, which may be a signature for a transition from a superfluid to a collisionless phase.
\end{abstract}

PACS numbers: 34.50.-s, 05.30.Fk, 39.25.+k, 32.80.Pj

The crossover from a Bose-Einstein condensate (BEC) to a Bardeen-Cooper-Schrieffer (BCS) superfluid has for decades attracted considerable attention in manybody theory [1]. Bose-Einstein condensates of molecules formed by fermionic atoms of ${ }^{6} \mathrm{Li}$ and ${ }^{40} \mathrm{~K}$ 2, 3, 3, 4, 5 provide a unique system to experimentally explore this BEC-BCS crossover. In such ultracold gases magnetically tuned scattering resonances, known as Feshbach resonances, allow to control and vary the interaction strength over a very broad range. Recent experiments have entered the crossover regime and yield results on the interaction strength by measuring the cloud size $[\underline{6}$ and expansion energy 5]. Moreover, two experiments 7, 8] have demonstrated the condensed nature of fermionic atom pairs in the crossover regime.

Important questions are related to superfluidity in the crossover regime [9]. When a molecular BEC is converted into an ultracold Fermi gas [6] , one can expect ultralow temperatures and superfluidity to extend far into the Fermi gas regime 10]. Detection tools to probe superfluidity in this regime are therefore requested. The investigation of collective excitation modes 11 is well established as a powerful method to gain insight into the physical behavior of ultracold quantum gases in different regimes of Bose 12] and Fermi gases [13. A recent paper 14 points out an interesting dependence of the collective frequencies in the BEC-BCS crossover of a superfluid Fermi gas. Superfluidity implies a hydrodynamic behavior which can cause substantial changes in the excitation spectrum and in general very low damping rates. However, in the crossover regime the strong interaction between the particles also results in hydrodynamic behavior in the normal, non-superfluid phase. Therefore the interpretation of collective modes in the BEC-BCS crossover in terms of superfluidity is not straightforward and needs careful investigation to identify the different regimes.

In this Letter, we report on measurements of fundamental collective excitation modes in the BEC-BCS crossover for various coupling strengths in the lowtemperature limit. In Ref. 2] we have already pre- sented a first measurement of the collective excitation of a molecular BEC in the limit of strong coupling. As described previously [2, 6] we work with a spin-mixture of ${ }^{6} \mathrm{Li}$ atoms in the two lowest internal states. For exploring different interaction regimes we use a broad Feshbach resonance, the position of which we determined to 837(5) G 15]. The different interaction regimes can be characterized by the coupling parameter $1 /\left(k_{F} a\right)$, where $a$ represents the atom-atom scattering length and $k_{F}$ is the Fermi wavenumber. Well below the Feshbach resonance $(B<700 \mathrm{G})$ we can realize the molecular BEC regime with $1 /\left(k_{F} a\right) \gg 1$. On resonance, we obtain the unitarity-limited regime of a universal fermionic quantum gas with $1 /\left(k_{F} a\right)=0$ [16]. An interacting Fermi gas of atoms is realized beyond the resonance where $1 /\left(k_{F} a\right)<0$.

The starting point of our experiments is a cigar-shaped molecular BEC produced by evaporative cooling in an optical dipole trap in the same way as described in Ref. []. Radially the sample is confined by a $35-\mathrm{mW}$ laser beam (wavelength $1030 \mathrm{~nm}$ ) focussed to a waist of $25 \mu \mathrm{m}$. The radial vibration frequency is $\omega_{r} \approx 2 \pi \times 750 \mathrm{~Hz}$. The axial vibration frequency is $\omega_{z}=2 \pi \times(601 B / \mathrm{kG}+11)^{1 / 2} \mathrm{~Hz}$, where the predominant contribution stems from magnetic confinement caused by the curvature of the Feshbach tuning field $B$ and a very small additional contribution arises from the weak axial optical trapping force.

For exploring collective excitations in the BEC-BCS crossover regime, we ramp the magnetic field from the evaporation field of $764 \mathrm{G}$, where the molecular BEC is formed, to fields between $676 \mathrm{G}$ and $1250 \mathrm{G}$ within one second. In previous work [6] we have shown that the conversion to an atomic Fermi gas proceeds in an adiabatic and reversible way, i.e. without increase of entropy. From the condensate fraction in the BEC limit, for which we measure more than $90 \%$ [6], we can give upper bounds for the temperature in both the BEC limit and the non-interacting Fermi gas limit of $T<0.46 T_{\mathrm{BEC}}$ and $T<0.03 T_{F}$ [10], respectively. Here $T_{\mathrm{BEC}}\left(T_{F}\right)$ denotes the critical temperature (Fermi temperature). With a total number of atoms $N \approx 4 \times 10^{5}$ (free atoms and atoms 


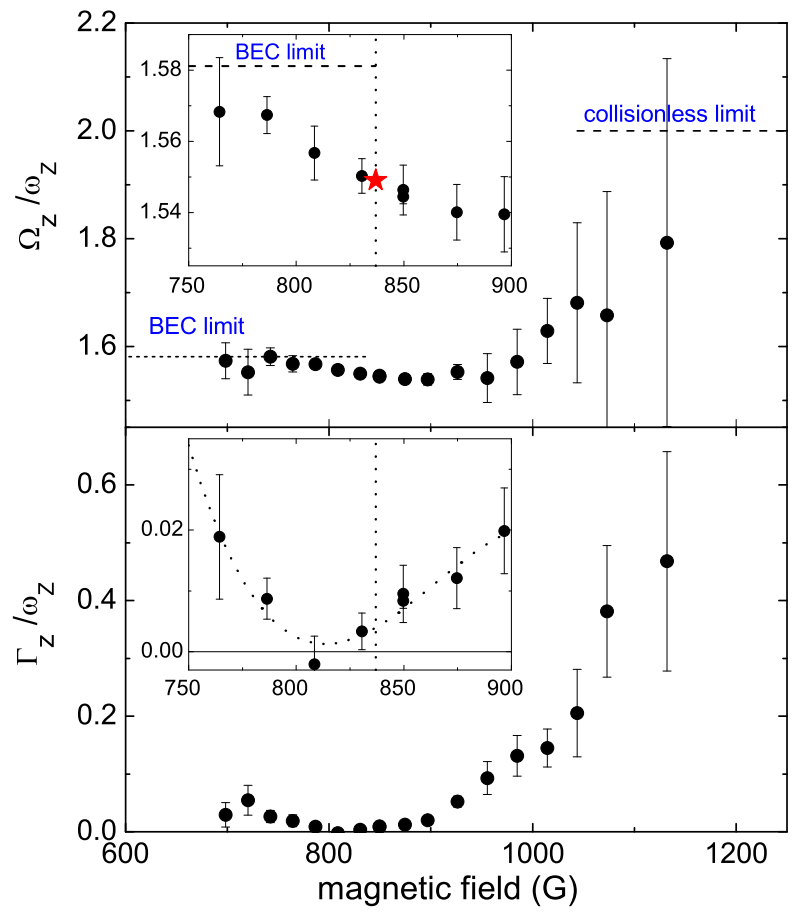

FIG. 1: Measured frequency $\Omega_{z}$ and damping rate $\Gamma_{z}$ of the axial compression mode, normalized to the trap frequency $\omega_{z}$. In the upper graph, the dashed lines indicate the BEC limit of $\Omega_{z} / \omega_{z}=\sqrt{5 / 2}$ and the collisionless Fermi gas limit with $\Omega_{z} / \omega_{z}=2$. The insets show the data in the resonance region. Here the vertical dotted line indicates the resonance position at $837(5) \mathrm{G}$. The star marks the theoretical prediction of $\Omega_{z} / \omega_{z}=\sqrt{12 / 5}$ in the unitarity limit. In the lower inset the dotted line is a third-order polynomial fit to the data.

bound to molecules) and a geometrically averaged trap frequency at $837 \mathrm{G}$ of $\bar{\omega}=\left(\omega_{r}^{2} \omega_{z}\right)^{1 / 3} \approx 2 \pi \times 230 \mathrm{~Hz}$, we calculate a Fermi energy $E_{F}=\hbar^{2} k_{F}^{2} / 2 m=\hbar \bar{\omega}(3 N)^{1 / 3}=$ $k_{B} \times 1.2 \mu \mathrm{K}$ for a non-interacting cloud, where $m$ is the mass of an atom and $k_{B}$ is Boltzmann's constant.

To excite the axial compression mode at a given magnetic field we increase the optical confinement in a 10ms time interval by a factor of 1.5. The laser power is varied slow enough for the radial motion to follow adiabatically, but fast enough to induce axial oscillations. The relative amplitude of the resulting axial oscillation is kept small, typically $\sim 10 \%$. We observe the oscillation by in-situ imaging of the cloud [6] after a variable hold time $t$ at constant trap parameters. To determine the collective oscillation frequency $\Omega_{z}$ and the damping rate $\Gamma_{z}$ we fit a damped harmonic oscillation $z(t)=z_{0}+A_{z} \exp \left(-\Gamma_{z} t\right) \sin \left(\Omega_{z} t+\phi_{z}\right)$ to the observed time evolution of the cloud size, where $z_{0}, A_{z}$, and $\phi_{z}$ are additional fit parameters.

The measured oscillation frequencies and damping rates are shown in Fig. 1. The data are normalized to the axial trap frequency $\omega_{z}$, as determined by excitation of the axial sloshing mode. We point out that the axial confinement is harmonic because of the dominant magnetic

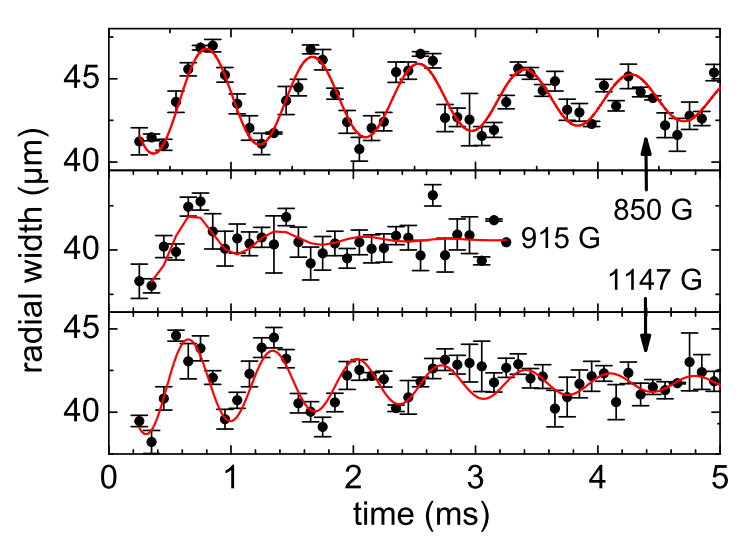

FIG. 2: Oscillations of the radial compression mode at different magnetic fields in the strongly interacting Fermi gas regime. The solid lines show fits by damped harmonic oscillations.

trapping and we can measure $\omega_{z}$ with a $10^{-3}$ precision. In the BEC limit, the measured collective frequencies are in agreement with the expected $\Omega_{z} / \omega_{z}=\sqrt{5 / 2}=1.581$ [11, 17]. With increasing magnetic field we observe a decrease in the collective excitation frequency until a minimum is reached at about $900 \mathrm{G}$, i.e. in the regime of a strongly interacting Fermi gas where $1 /\left(k_{F} a\right) \approx-0.5$. With further increasing magnetic field and decreasing interaction strength, we then observe a gradual increase of the collective frequency toward $\Omega_{z} / \omega_{z}=2$. The latter value is expected for a collisionless degenerate Fermi gas, where the elastic collision rate is strongly reduced by Pauli blocking. Because of the large damping rates in the transition regime between hydrodynamic and collisionless behavior, the excitation frequencies cannot be determined with high accuracy. The observed axial damping is consistent with a gradual transition between these two regimes [18].

The insets in Fig. 1 show a zoom-in of the data for the resonance region between 750 and $900 \mathrm{G}$. The collective frequency that we measure on resonance exhibits the small $2 \%$ down shift expected for the unitarity limit $\left(\Omega_{z} / \omega_{z}=\sqrt{12 / 5}=1.549\right)[14]$. For the damping rate we observe a clear minimum at a magnetic field of $815(10) \mathrm{G}$, which is remarkably close to the resonance position. It is interesting to note that this damping minimum coincides with the recent observation of a maximum fraction of condensed fermionic atom pairs in Ref. [8]. For the minimum damping rate we obtain the very low value of $\Gamma_{z} / \omega_{z} \approx 0.0015$, which corresponds to a $1 / e$ damping time of $\sim 5 \mathrm{~s}$.

To excite the radial compression mode we reduce the optical confinement for $50 \mu \mathrm{s}$, which is short compared with the radial oscillation period of $1.3 \mathrm{~ms}$. In this short interval the cloud slightly expands radially, and then begins to oscillate when the trap is switched back to the initial laser power. The relative oscillation amplitude is $\sim 10 \%$. To detect the radial oscillation, we 


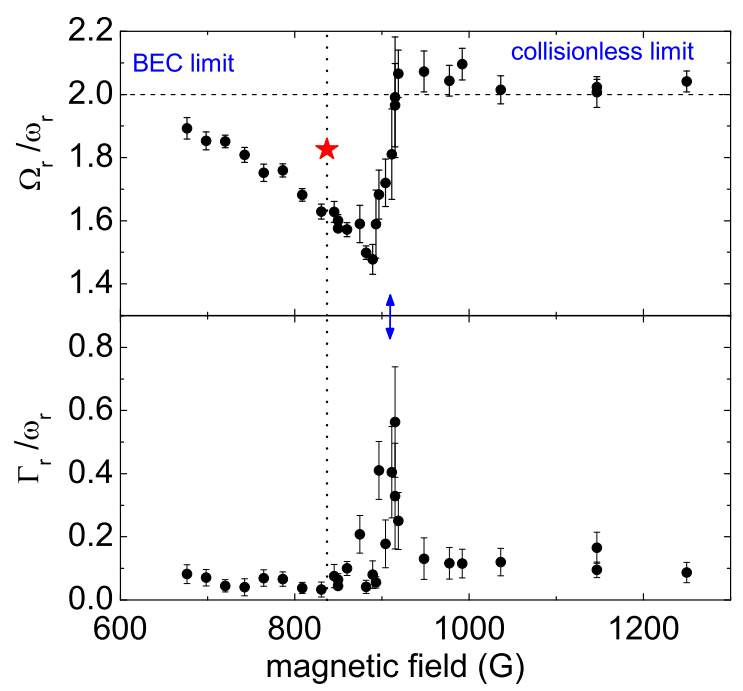

FIG. 3: Measured frequency $\Omega_{r}$ and damping rate $\Gamma_{r}$ of the radial compression mode, normalized to the trap frequency (sloshing mode frequency) $\omega_{r}$. In the upper graph, the dashed line indicates $\Omega_{r} / \omega_{r}=2$, which corresponds to both the BEC limit and the collisionless Fermi gas limit. The vertical dotted line marks the resonance position at $837(5) \mathrm{G}$. The star indicates the theoretical expectation of $\Omega_{r} / \omega_{r}=\sqrt{10 / 3}$ in the unitarity limit. A striking change in the excitation frequency occurs at $\sim 910 \mathrm{G}$ (arrow) and is accompanied by anomalously strong damping.

turn off the trapping laser after various delay times $t$ and measure the radial size $r(t)$ after $1.5 \mathrm{~ms}$ of expansion. The measured radial size $r(t)$ reflects the oscillating release energy. From the corresponding experimental data, we extract the excitation frequency $\Omega_{r}$ and damping $\Gamma_{r}$ by fitting the radial cloud size to $r(t)=$ $r_{0}+A_{r} \exp \left(-\Gamma_{r} t\right) \sin \left(\Omega_{r} t+\phi_{r}\right)$, where $r_{0}, A_{r}$ and $\phi_{r}$ are additional fit parameters. Typical radial oscillation curves are shown in Fig. 2

The magnetic-field dependence of the radial excitation frequency $\Omega_{r}$ and the damping rate $\Gamma_{r}$ is shown in Fig. 3. Here we normalize the data to the trap frequency $\omega_{r}$, which we obtain by measuring the radial sloshing mode at the given magnetic field [19]. This normalization suppresses anharmonicity effects in the measured compression mode frequency to below $3 \%$ 21]. For low magnetic fields, the measured frequency ratio approaches the BEC limit 11, 20] $\left(\Omega_{r} / \omega_{r}=2\right)$. With increasing magnetic field, i.e. increasing interaction strength, we observe a large down-shift of the frequency. On resonance $(B=837 \mathrm{G})$, we observe $\Omega_{r} / \omega_{r}=1.62(2)$. Above resonance, i.e. with the gas entering the strongly interacting Fermi gas regime, the oscillation frequency further decreases until a maximum shift of almost $30 \%$ $\left(\Omega_{r} / \omega_{r}=1.42(5)\right)$ is reached at $B=890 \mathrm{G}$. With further increasing magnetic field, i.e. decreasing interaction strength, an abrupt change to $\Omega_{r} / \omega_{r} \approx 2$ is observed. For $B>920 \mathrm{G}$ our data are consistent with a Fermi gas in the collisionless regime. The damping of the radial compression mode is small in the BEC limit and reaches a minimum close to the unitarity regime. At $B=910 \mathrm{G}$, where the abrupt change occurs, we observe very strong damping (see also middle trace in Fig. 21).

We have performed further experiments to check our data on the radial compression mode for systematic effects. We have repeated the measurements after recompressing the trap to 9 times higher trap laser power $\left(\omega_{r} \approx 2.4 \mathrm{kHz}\right)$. The corresponding data confirm all our observations in the shallower trap. In particular, the negative frequency shift and the sudden change in the collective frequency show up in essentially the same way. The recompressed trap also allows us to eliminate a small residual anharmonicity shift from our measurement of the collective frequency at $837 \mathrm{G}$, and we obtain $\Omega_{r} / \omega_{r}=1.67(3)$ for the harmonic trap limit. We have also checked that the frequency of the compression mode in the resonance region does not depend on the way we prepare the ultracold gas. Direct evaporation at a fixed magnetic field, without starting from a molecular BEC, leads to the same collective frequency. Preliminary measurements at higher temperatures, however, show a trend towards smaller frequency shifts in the radial compression mode and to smoother changes of the collective frequency.

Our measurements on the radial compression mode show three surprises. The corresponding features, which we discuss in the following, cannot be explained on the basis of available theoretical models and suggest new physics in the BEC-BCS crossover regime.

Surprise one: For a strongly interacting BEC, Ref. 22] has predicted up-shifts of the collective frequencies with increasing coupling strength based on beyond mean-field theory corrections 23]. Applying these predictions to a molecular BEC in the crossover regime, the collective excitation frequencies should follow $\delta \Omega_{i} / \Omega_{i}=c_{i} \sqrt{n_{m} a_{m}^{3}}$ $(i=z, r)$, where $n_{m}$ is the peak molecular number density and $a_{m}=0.6 a[24]$ is the molecule-molecule scattering length. For our highly elongated trap geometry the numerical factors are $c_{r}=5 c_{z}=0.727$. In contrast to these expectations, we observe a strong frequency downshift in the radial direction. Using the above formula to fit the first four data points, we obtain a negative coefficient of $c_{r}=-1.2(3)$. For the axial oscillation we obtain $c_{z}=-0.04(5)$. Note that a substantial down-shift in radial direction is observed even at the low magnetic field of $676 \mathrm{G}$ where the molecular gas parameter is relatively small $\left(n_{m} a_{m}^{3}=0.001\right)$. Apparently, the beyond mean field theory of a BEC is not adequate to describe the transition from a molecular BEC to a strongly interacting gas in the BEC-BCS crossover.

Surprise two: The universal character of the strongly interacting quantum gas on resonance suggests a simple equation of state for which one expects $\Omega_{z} / \omega_{z}=$ $\sqrt{12 / 5}=1.549$ and $\Omega_{r} / \omega_{r}=\sqrt{10 / 3}=1.826$ for the collective excitation frequencies [14]. While our measurements confirm the predicted axial frequency, we obtain a different frequency in the radial direction of $\Omega_{r} / \omega_{r}=$ 
$1.67(3)$.

Surprise three: The abrupt change of the excitation frequency and the large damping rate are not expected in a normal degenerate Fermi gas, where the collective excitation frequency is expected to vary smoothly from the hydrodynamic regime to the collisionless one. Furthermore, for the damping rate of the radial mode in the transition regime a maximum value of $\Gamma_{r} / \omega_{r}=0.09$ is calculated in Ref. 18]. Our measured damping rate of $\Gamma_{r} / \omega_{r} \approx 0.5$ is clearly inconsistent with this prediction for the normal (non-superfluid) hydrodynamic regime. However, both the sudden change of the collective frequency and a strong damping are expected for a transition from the superfluid to the normal phase [25].

In conclusion, our experiments demonstrate that the collective modes of a degenerate gas in the BEC-BCS crossover region show a pronounced dependence on the coupling strength and thus provide valuable information on the physical behavior of the system. For the axial compression mode, the frequency shift observed in the unitarity limit confirms theoretical expectations. However, the radial compression mode reveals a surprising behavior. In the strongly interacting BEC regime, the observed frequency shifts have an opposite sign as compared to expectations from beyond mean field theory and the frequency shift on resonance is even larger than ex- pected. The most striking feature is an abrupt change of the radial collective frequency in the regime of a strongly attractive Fermi gas where $1 /\left(k_{F} a\right) \approx-0.5$. The transition is accompanied by very strong damping. The observation supports an interpretation in terms of a transition from a hydrodynamic to a collisionless phase. A superfluid scenario for the hydrodynamic case seems plausible in view of current theories on resonance superfluidity [9] and the very low temperatures provided by the molecular BEC [10]. A definite answer, however, to the sensitive question of superfluidity requires further careful investigations, e.g. on the temperature dependence of the phase transition.

We warmly thank S. Stringari for stimulating this work and for many useful discussions. We also thank W. Zwerger and M. Baranov for very useful discussions. We acknowledge support by the Austrian Science Fund (FWF) within SFB 15 (project part 15) and by the European Union in the frame of the Cold Molecules TMR Network under Contract No. HPRN-CT-2002-00290. C.C. is a Lise-Meitner research fellow of the FWF.

Note added: A recent paper by John Thomas's group [26] reports on measurements of the radial compression mode in the resonance region, which show much weaker frequency shifts than we observe. This apparent discrepancy needs further investigation.
[1] A.J. Leggett, in Modern Trends in the Theory of Condensed Matter, edited by A. Pekalski and R. Przystawa (Springer-Verlag, Berlin, 1980); P. Nozières and S. Schmitt-Rink, J. Low Temp. Phys. 59, 195 (1985); E. Timmermans, K. Furuya, P.W. Milonni, A.K. Kerman, Phys. Lett. A 285, 228 (2001); J.N. Milstein, S.J.J.M.F. Kokkelmans, and M. Holland, Phys. Rev. A 66, 043604 (2002); Y. Ohashi, A. Griffin, Phys. Rev. Lett. 89, 130402 (2002); R. Combescot, Phys. Rev. Lett. 91, 120401 (2003); A. Perali, P. Pieri, and G.C. Strinati, Phys. Rev. A 68, 031601(R) (2003); J. Stajic et al., cond-mat/0309329

[2] S. Jochim et al., Science 302, 2101 (2003).

[3] M. Greiner, C.A. Regal, and D.S. Jin, Nature 426, 537 (2003).

[4] M. W. Zwierlein et al., Phys. Rev. Lett. 91, 250401 (2003).

[5] T. Bourdel et al., cond-mat/0403091

[6] M. Bartenstein et al., Phys. Rev. Lett. 92, 120401 (2004).

[7] C.A. Regal, M. Greiner, and D.S. Jin, Phys. Rev. Lett. 92, 040403 (2004).

[8] M.W. Zwierlein et al., Phys. Rev. Lett. 92, 120403 (2004).

[9] M. Holland et al., Phys. Rev. Lett. 87, 120406 (2001).

[10] L.D. Carr, G.V. Shlyapnikov, and Y. Castin, Phys. Rev. Lett. 92, 150404 (2004).

[11] S. Stringari, Phys. Rev. Lett. 77, 2360 (1996).

[12] L. Pitaevski and S. Stringari, Bose-Einstein Condensation (Clarendon Press, Oxford, 2003), and refs. therein.

[13] G. M. Bruun and C.W. Clark, Phys. Rev. Lett. 83, 5415 (1999); G. M. Bruun and B. R. Mottelson, Phys. Rev.
Lett. 87, 270403 (2001); A. Minguzzi and M. P. Tosi, Phys. Rev. A 63, 023609 (2001).

[14] S. Stringari, Europhys. Lett. 65, 749 (2004).

[15] We determined the resonance position by measuring the molecular binding energy using radio-frequency spectroscopy as introduced in C.A. Regal, C. Ticknor, J.L. Bohn, and D.S. Jin, Nature 424, 47 (2003).

[16] H. Heiselberg, Phys. Rev. A 63, 043606 (2001).

[17] M.-O. Mewes et al., Phys. Rev. Lett. 77, 988 (1996).

[18] L. Vichi, J. Low Temp. Phys. 121, 177 (2000).

[19] We excite the radial sloshing mode by pulsed application of a vertical magnetic gradient. With increasing magnetic field and thus increasing radial cloud size, the anharmonicity of the Gaussian-shaped radial potential leads to a decrease of the sloshing mode frequency. For $\omega_{r} / 2 \pi$ we observe a corresponding decrease from $765 \mathrm{~Hz}$ at $676 \mathrm{G}$ to $710 \mathrm{~Hz}$ at magnetic fields above $1.2 \mathrm{kG}$; at $837 \mathrm{G}$ we obtain $740 \mathrm{~Hz}$. Our measurements of the sloshing mode frequency agree well with an expected scaling of the lowestorder anharmonicity correction with the squared cloud size [27].

[20] F. Chevy et al., Phys. Rev. Lett. 88, 250402 (2002).

[21] The anharmonicity corrections to frequencies of both the compression mode $\left(\Omega_{r}\right)$ and the sloshing mode $\left(\omega_{r}\right)$ scale in the same way with the increasing cloud size with a slightly larger prefactor for the compression mode [27]. Our normalization $\Omega_{r} / \omega_{r}$ reduces the anharmonicity effect by typically a factor of five.

[22] L. Pitaevski and S. Stringari, Phys. Rev. Lett. 81, 4541 (1998).

[23] T. D. Lee, K. W. Huang, and C. N. Yang, Phys. Rev. 
106, 1135 (1957).

[24] D.S. Petrov, C. Salomon, G.V. Shlyapnikov, cond-mat/0309010

[25] M. A. Baranov and D. S. Petrov, Phys. Rev. A 62, 041601

(2000).

[26] J. Kinast et al., Phys. Rev. Lett. 92, 150402 (2004).

[27] S. Stringari, private communication. 